\title{
SPECTROMÈTRE MULTICANAL NUMÉRIQUE POUR LA MESURE DE LA TEMPÉRATURE IONIQUE D'UN PLASMA RÉCURRENT
}

\author{
M. CLÉMENT, B. MOULIN et P. STEVENIN \\ Service d'Ionique Générale. Centre d'Etudes Nucléaires de Grenoble \\ BP 85, Grenoble 38041, France
}

(Reçu le 20 novembre 1973, révisé le 23 janvier 1973)

\begin{abstract}
Résumé. - On décrit un spectromètre multicanal numérique construit pour la mesure de la température ionique d'un plasma récurrent.

Le choix du disperseur interférentiel Fabry-Pérot, de 12 voies spectrales et du système numérique d'acquisition et de présentation des résultats s'est imposé à cause de l'extrême sensibilité demandée (plasma peu lumineux) et de la grande souplesse d'emploi de l'électronique numérique.

L'erreur sur la mesure de la température ionique donnée par un spectromètre multicanal est étudiée et est comparée à celle trouvée expérimentalement après lissage des profils enregistrés. Enfin on donne les premiers résultats obtenus sur l'expérience CIRCE $25 \mathrm{~kW}$.
\end{abstract}

\begin{abstract}
A multichannel digital spectrometer has been designed for the evaluation of the ionic temperature in recurrent plasma.

The Fabry-Perot interferometric disperser, the 12 spectral channels, and the digital system for storage and output of the results were chosen because of the high sensitivity requirements (very weak light from the plasma) and the flexibility of the digital electronics. The problem of error given by a multichannel spectrometer is discussed and compared to the experimental results obtained after smoothing. Finally, we present the first results of measurements from the CIRCE $25 \mathrm{~kW}$ device.
\end{abstract}

1. Introduction. - La mesure de la température ionique d'un plasma par la méthode optique de l'élargissement Doppler des raies d'émission a déjà été faite par différentes méthodes [1-2-3]. La nature de la décharge peut dicter le choix de l'instrument de mesure et du système de détection. Dans le cas d'un plasma très peu lumineux, un appareil de grande luminosité est nécessaire et alors le choix de l'interféromètre Fabry-Pérot comme élément dispersif s'impose [4]. $\mathrm{Si}$, en outre, le plasma est récurrent, une accumulation de photoélectrons pendant un certain temps suivant une technique de comptage, permettra d'enregistrer un signal significatif. La grande souplesse de cette technique, dans le cas d'un plasma récurrent et reproductible, facilite aussi la résolution temporelle. Mais, les temps de pose pouvant devenir longs, on doit assurer une grande stabilité à l'appareillage et éliminer toutes impulsions parasites qui risquent de se superposer et même de noyer celles qui proviennent de la raie à analyser. Différents auteurs ont déjà utilisé avec profit les méthodes de comptage photonique pour la spectrométrie des lumières faibles [5-6-7-8].

Nous décrirons tout d'abord le spectromètre multicanal, en insistant particulièrement sur le système d'acquisition numérique. Puis, nous étudierons dans le cadre de la mesure de la température ionique, la précision de la mesure et l'apport du lissage des profils. Enfin nous donnerons les résultats obtenus avec cet appareil sur un plasma récurrent de notre laboratoire.

2. Le spectromètre multicanal. -2.1 DesCRIPTION DU MONTAGE OPTIQUE. - Le spectromètre interférentiel multicanal (quelquefois appelé FAFNIR) a déjà été décrit dans différents articles [3-8]. On rappelle simplement que la pièce essentielle est un séparateur spectral constitué de 12 miroirs annulaires, concentriques et de surfaces égales, qui distribue sur 12 récepteurs photoélectriques la lumière provenant de 12 éléments spectraux qui constituent la raie.

Le spectromètre est schématisé sur la figure 1 . Le filtre interférentiel qui isole une bande spectrale autour de la raie à étudier, permet de conserver l'étendue géométrique permise par l'emploi de l'étalon Fabry-Pérot. Ce dernier constitue le disperseur spectral $\mathrm{du}$ spectromètre. Un télé-objectif de focale $\mathrm{F}$ forme l'image du premier anneau d'interférence sur les 12 miroirs. Il est à noter que le spectromètre a été conçu à l'origine pour l'enregistrement "simultané " 


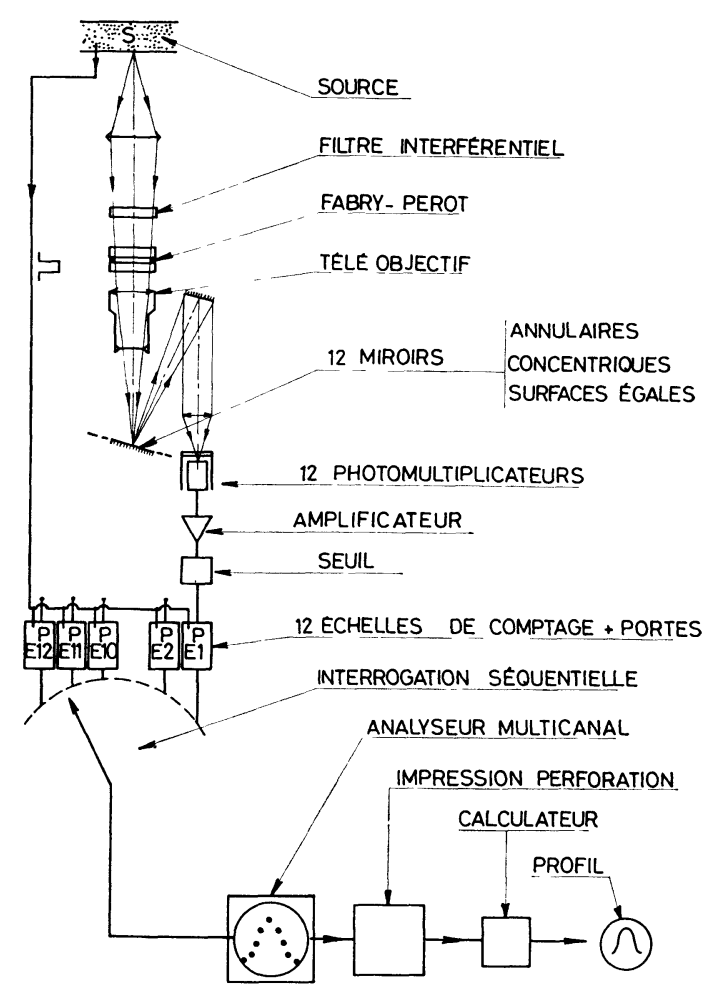

FIG. 1. - Schéma optique et électronique du spectromètre multicanal digital.

de 12 signaux photoélectriques, permettant de reconstituer plusieurs profils à partir d'une seule décharge [3]. Ici on le détourne de sa première utilisation car on accumule un très grand nombre d'impulsions provenant de plusieurs décharges successives. Il est évident que le principal avantage de notre spectromètre par rapport à un spectromètre à défilement lent mais de résolution et de luminosité équivalentes, est de gagner un facteur 12 sur le temps de pose.

La lumière ainsi décomposée tombe sur 12 photomultiplicateurs à très faible bruit d'obscurité de E. M. I. (type $6256 \mathrm{SA}$ ) qui peuvent être éventuellement refroidis par une circulation d'azote gazeux à $-10^{\circ} \mathrm{C}$.

2.2 LE SYSTÈME DE DÉTECTION. - La chaîne d'acquisition numérique se compose d'éléments classiques :

- Derrière chaque photomultiplicateur, nous avons placé un amplificateur d'impulsion avec un gain de 100 , une bande passante de $10 \mathrm{MHz}$ et un niveau de sortie élevé $(10 \mathrm{~V})$ pour tenir compte non seulement de l'affaiblissement en ligne coaxiale ( $30 \mathrm{~m}$ séparent le spectromètre du pupitre de mesure) mais aussi du niveau du seuil.

- Les seuils de chaque voie ont été placés à $1 \mathrm{~V}$. Une étude préalable des photomultiplicateurs utilisés a montré en effet que la croissance du rapport signal/ bruit est faible ou nulle pour des valeurs de seuil au-dessous de $1 \mathrm{~V}$ (voir Fig. 2).

- Les 12 échelles de comptage sont limitées à $10 \mathrm{MHz}$ et le temps mort mesuré est d'environ $100 \mathrm{~ns}$.

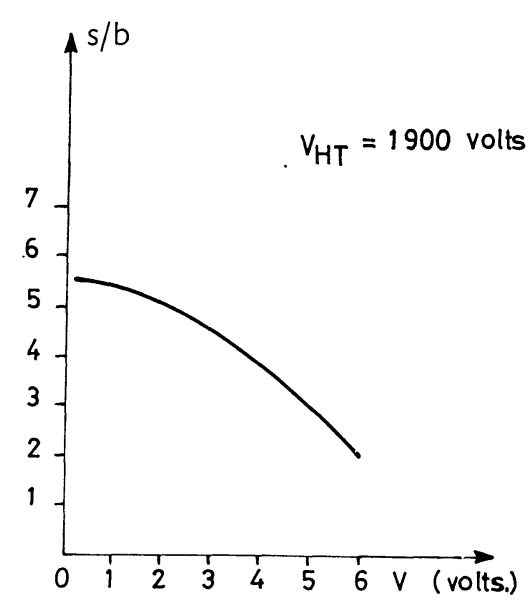

Fig. 2. - Variation du rapport signal/bruit en fonction de la position du seuil.

Ceci assure cependant une dynamique photométrique très élevée. En effet, il est possible à la fois de détecter des lumières donnant moins d'une impulsion par seconde et de compter à $1 \%$ près jusqu'à $10^{5}$ impulsions/s.

Le temps d'ouverture de ces échelles (ou portes) est ajustable ; elles sont déclenchées par le top de synchronisation du plasma suivi d'un retard également ajustable. Le réglage de ces durées (cycle, pose, retard) est obtenu par précompte d'impulsions horloge.

2.3 L'UNITÉ LOGIQUE DE COMMANDE. - Nous avons développé un système très souple permettant de traiter aussi bien les informations optiques entièrement numériques et les diverses valeurs analogiques utiles au réglage et à l'exploitation de cet ensemble spectrométrique.

Comme on le voit sur la figure 3 , une unité logique de commande (programme sur carte matrice à diodes) détermine la séquence des adresses et des instructions de manière que les différentes mesures analogiques ou numériques soient aiguillées convenablement et en temps voulu. D'une façon plus précise, on dispose des 4 programmes suivants:

a) Programme de relevé cyclique du contenu des 12 échelles et de son transfert dans un analyseur multicanal permettant une visualisation du profil. Cette opération étant exécutée avec ou sans remise à zéro des échelles, on peut soit suivre l'évolution de la raie spectrale, soit prolonger la pose récurrente pour améliorer le rapport $\mathrm{s} / \mathrm{b}$ du profil, à condition naturellement que le plasma récurrent soit stable.

b) Programme du relevé cyclique ou commandé de 16 informations analogiques (seuil, température de l'enceinte contenant l'étalon Fabry-Pérot, tension, etc.). Chacune d'elles, après passage dans un codeur (voltmètre numérique avec affichage) est imprimée et perforée. Cette petite centralisation des mesures facilite grandement les réglages, puis la vérification des calages corrects des paramètres essentiels. 


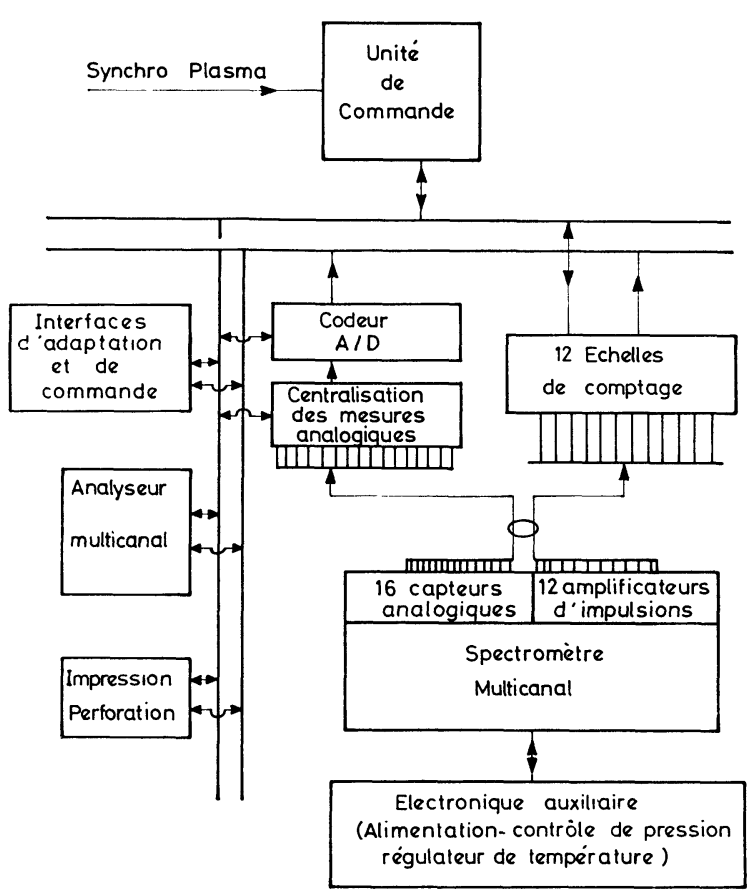

FIG. 3. - Diagramme de l'unité logique de commande.

c) Programme du relevé cyclique du contenu d'une échelle particulière, et de son transfert dans la mémoire de l'analyseur et visualisation «multi-échelle ». Cette fonction sert en particulier au réglage périodique du centrage, et de la finesse de l'interféromètre.

d) Commande d'impression et de perforation sur ruban des valeurs mémorisées dans l'analyseur multicanal.

2.4 RÉGlages. Stabilité. - Le réglage optique essentiel consiste à centrer l'anneau d'interférence sur les miroirs annulaires. Ce réglage se fait à l'aide d'une raie fine. Un balayage par pression de l'interféromètre amène le maximum de la raie sur le canal désiré (6 ou 7), et le centrage est obtenu par inclinaison du F. P. à l'aide de 3 vis calantes.

Si l'on désire un profil correct, il faut évidemment qu'une lumière d'énergie spectrale constante (du moins sur plusieurs intervalles spectraux libres) donne sur chacune des 12 voies, un signal de même amplitude. L'équilibrage de ces 12 voies s'obtient en jouant sur les tensions anodiques de 11 photomultiplicateurs pour que leur fréquence de comptage relative à une faible lumière (soit $10^{4} \mathrm{cps} / \mathrm{s}$ ) soit la même que celle d'un photomultiplicateur dont on a optimisé les paramètres $(V=1900 \mathrm{~V})$.

Il est à noter que cet équilibrage des voies doit être fait à la température de fonctionnement des photomultiplicateurs (généralement $-10^{\circ} \mathrm{C}$ ).

Cet équilibrage se conserve plusieurs jours, et on a remarqué que le vieillissement des photomultiplicateurs améliore la stabilité différentielle dans le temps.
3. Recherche de l'utilisation optimum du spectromètre multicanal. - Puisque le plasma est récurrent et reproductible, les impulsions photoélectriques reçues dans chaque canal sont accumulées pendant un temps suffisamment long de façon à avoir un signal significatif.

La précision de la mesure est naturellement reliée au temps de pose. Afin de réduire ce temps de pose, il nous a paru intéressant de rechercher une optimisation de l'emploi du spectromètre multicanal.

Le problème est donc de choisir, pour un nombre $Q$ de photoélectrons représentatifs de tout le profil spectral, le meilleur calage instrumental, de façon à avoir une erreur relative minimale sur la largeur du profil, donc sur la température ionique à mesurer.

Nous avons supposé que le profil était purement gaussien. Le nombre de photoélectrons émis par la photocathode dans l'intervalle spectral $\Delta \lambda$ autour de $\lambda$ est alors donné par:

$$
n \Delta \lambda=n_{0} \exp \left\{-\frac{M c^{2}}{2 k T_{\mathrm{i}}}\left(\frac{\lambda-\lambda_{0}}{\lambda_{0}}\right)^{2}\right\} \Delta \lambda
$$

où $n_{0} \Delta \lambda$ est le nombre de photoélectrons autour de $\lambda_{0}$ dans le canal central ; $M, c, k$ sont respectivement la masse de l'ion émetteur, la vitesse de la lumière, et la constante de Boltzmann, et $T_{\mathrm{i}}$ est la température ionique à mesurer.

Un calcul simple montre que l'erreur relative sur $T_{\mathrm{i}}$ s'exprime par une somme de deux termes positifs :

$$
\frac{\mathrm{d} T_{\mathrm{i}}}{T_{\mathrm{i}}}=\frac{1}{\log \left(n_{0}\right)-\log (n)}\left\{\frac{\mathrm{d} n_{0}}{n_{0}}+\frac{\mathrm{d} n}{n}\right\}+\frac{2 \mathrm{~d} \lambda}{\lambda-\lambda_{0}} .
$$

Physiquement l'expression entre parenthèse provient de 2 causes d'erreurs :

- l'erreur sur l'ordonnée $n$ due à la fluctuation statistique des photoélectrons contenus dans le canal de largeur $\Delta \lambda$, et qui est proportionnelle à $\mu \sqrt{n \Delta \lambda}$ (le coefficient $\mu$ qui représente l'écart à la loi de Poisson a été analysé systématiquement et a été trouvé voisin de 1 à $5 \%$ près [9]).

On pose

$$
n=n_{0} \exp \left(-W^{2}\right) \text { avec } W=\left(\lambda-\lambda_{0}\right) / \Delta \lambda_{e}
$$

et

$$
\Delta \lambda_{e}=\lambda_{0}\left(\frac{2 k T_{\mathrm{i}}}{M c^{2}}\right)^{1 / 2}
$$

$\Delta \lambda_{e}$ est la demi-largeur de la raie à $1 / e$ de l'intensité maximum. On a donc :

$$
\frac{\mathrm{d} n}{n}=\frac{\mu}{(n \Delta \lambda)^{1 / 2}} \simeq \frac{1}{\left(n_{0} \Delta \lambda\right)^{1 / 2}} \exp \left(\frac{W^{2}}{2}\right)
$$

de même :

$$
\frac{\mathrm{d} n_{0}}{n_{0}} \simeq \frac{1}{\left(n_{0} \Delta \lambda\right)^{1 / 2}}
$$


- L'erreur sur l'abscisse $\lambda$ due à la limite de résolution du spectromètre se calcule aisément à partir de la dérivée logarithmique :

$$
\frac{\mathrm{d} n_{0}}{n_{0}}+\frac{\mathrm{d} n}{n}=-2 W \mathrm{~d} W=-2 W \frac{\mathrm{d} \lambda}{\Delta \lambda_{e}} .
$$

Finalement la récapitulation de (3), (4), (5) dans donne :

$$
\begin{aligned}
\frac{\mathrm{d} T_{\mathrm{i}}}{T_{\mathrm{i}}}=\left[\frac{1+\exp \left(W^{2} / 2\right)}{W^{2}}\right] & \times \frac{1}{\left(n_{0} \Delta \lambda\right)^{1 / 2}}+ \\
& +2\left(W+\frac{1}{W}\right) \frac{\mathrm{d} \lambda}{\Delta \lambda_{e}} .
\end{aligned}
$$

Introduisons le nombre total $Q$ de photoélectrons dans la raie gaussienne, soit :

$$
Q=n_{0}(\pi)^{1 / 2} \Delta \lambda_{e}
$$

$\mathrm{d} \lambda$ est une fraction $\beta(0 \leqslant \beta \leqslant 1)$ de la largeur d'un canal $\Delta \lambda$ :

$$
\mathrm{d} \lambda=\beta \Delta \lambda
$$

Si $p$ est le nombre de canaux constituant le multicanal, et si $\lambda_{L}$ est la longueur d'onde correspondant

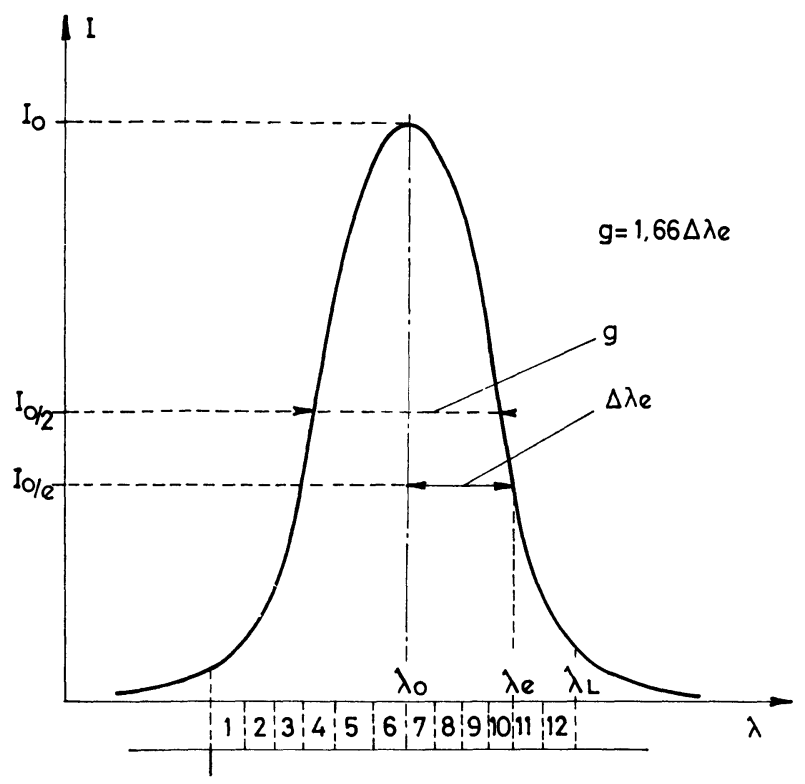

FIG. 4. - Centrage d'une raie gaussienne à $1,5 \Delta \lambda_{e}$ sur le multicanal.

à l'extrémité du $p$-ième canal, nous posons (voir Fig. 4) :

$$
u=\left|\lambda_{L}-\lambda_{0}\right| / \Delta \lambda_{e}
$$

et

$$
2 u \Delta \lambda_{e}=p \Delta \lambda \text {. }
$$

L'expression finale de l'erreur relative sur $T_{\mathrm{i}}$ s'écrit donc à partir de la relation (6) :

$$
\begin{aligned}
\frac{\mathrm{d} T_{\mathrm{i}}}{T_{\mathrm{i}}}=\left[\frac{1+\exp \left(\frac{W^{2}}{2}\right)}{W^{2}}\right](\pi)^{1 / 4}\left(\frac{p}{2 u Q}\right)^{1 / 2}+ \\
+\frac{4 \beta u}{p}\left(W+\frac{1}{W}\right) .
\end{aligned}
$$

Pour la recherche du meilleur calage instrumental, nous avons tracé les courbes $5 a$ en fonction de $W$ et $5 b$ en fonction de $u$ pour 12 canaux $(p=12)$. Compte tenu de ce que $u$ doit être à peine supérieur à $W$ à cause du second terme de (7), on a choisi le calage instrumental suivant: $W=1, u=1,5$. Ainsi la précision obtenue sera optimum lorsque la largeur mesurée est la largeur prise à $1 / e$ de l'intensité maximum $(W=1)$ et que le domaine spectral nécessaire pour analyser la raie contient 3 fois la demi-largeur $\Delta \lambda_{e}(u=1,5)$.
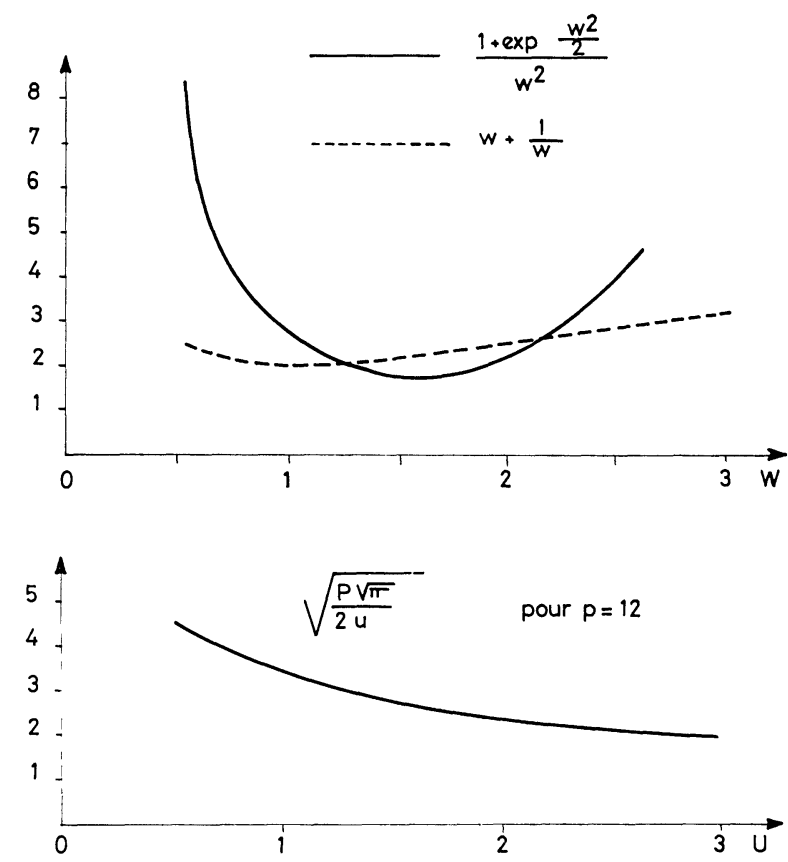

Fig. $5 a, 5 b$. - Courbes d'optimisation en fonction de $W$ et de $u$.

Si nous posons $\beta=0,3$ (ce qui est raisonnable si on veut négliger la fonction instrumentale) l'égalité (7) devient :

$$
\frac{\mathrm{d} T_{\mathrm{i}}}{T_{\mathrm{i}}}=\frac{7,5}{Q^{1 / 2}}+0,3
$$

On fait donc une erreur sur $T_{\mathrm{i}}$ voisine de $50 \%$ lorsqu'il y a environ 1500 photoélectrons dans la raie spectrale.

Malgré ce calage optimum on voit que l'erreur relative sur la mesure sera toujours supérieure à $30 \%$ quel que soit le nombre de photoélectrons $Q$. 
Mais cette précision peut sensiblement être améliorée lorsque l'on utilise un lissage du profil physique à partir d'une fonction mathématique connue. Physiquement, cela se conçoit aisément puisque les $p$ signaux contribuent (il est vrai d'une manière inégale) à la détermination finale de la largeur de la raie.

Pour apprécier le gain apporté par le lissage, nous avons éclairé le spectromètre avec une raie de l'hélium en respectant les conditions d'optimisation fixées ci-dessus $(u=1,5, W=1, \beta=0,3)$. Puis on a enregistré une série de profils d'intensité décroissante.

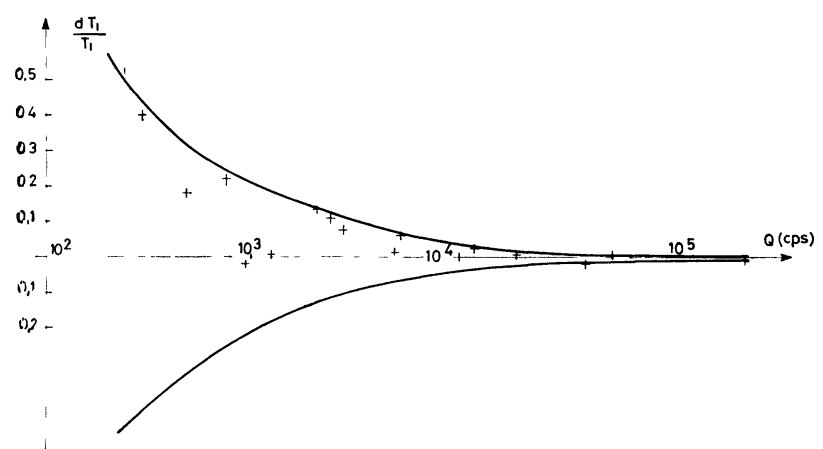

FIG. 6. - Erreur relative sur $T_{\mathrm{i}}$ après lissage en fonction du nombre d'impulsions $Q$ dans la raie.

On a tracé sur la figure 6 la précision relative sur $T_{\mathrm{i}}$ à partir de la dispersion des largeurs $\Delta \lambda_{e}$ calculées après lissage avec naturellement :

$$
\frac{\mathrm{d} T_{\mathrm{i}}}{T_{\mathrm{i}}}=\frac{2 \mathrm{~d}\left(\Delta \lambda_{e}\right)}{\Delta \lambda_{e}} .
$$

En abscisse $Q$ est le nombre total de photoélectrons après avoir retranché canal par canal, le bruit d'obscurité du signal.

On voit que la précision ainsi mesurée est bien supérieure à la précédente $\mathrm{d} T_{\mathrm{i}} / T_{\mathrm{i}} \simeq \pm 20 \%$ pour $Q=1000 \mathrm{imp} / \mathrm{s}$. En particulier le terme 0,3 de l'expression (8), est remplacé par un terme 10 fois plus faible.

Remarques. $-1^{\circ}$ Le choix des 12 canaux pouvant paraitre arbitraire, on a recherché l'importance du facteur $p$ sur la précision de la température. Utilisant la relation [7] avec $W=1, u=1,5, \beta=0,3$ on a porté sur la figure 7 la précision $\mathrm{d} T_{\mathrm{i}} / T_{\mathrm{i}}$ en fonction de $p$ pour 2 valeurs de $Q$.

On constate comme on devait s'y attendre que pour les fortes lumières, la précision s'améliore avec le nombre de canaux, tandis qu'elle passe par un optimum pour les lumières très faibles. Dans ce dernier cas, la précision varie peu de $p=6$ à $p=60$, et cela justifie a posteriori le choix de 12 canaux.

$2^{\circ}$ Pour tenir compte d'une réalité plus physique, le calage instrumental devra être modifié lorsqu'on enregistre une raie en présence d'un fond continu non négligeable. On a observé que dans le cas du lissage, la valeur prise $u=1,5$ parait trop faible. En

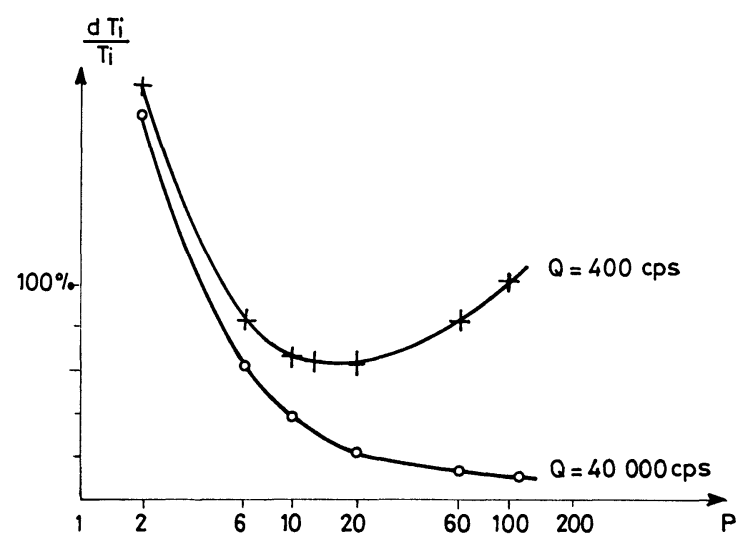

Fig. 7. - Précision relative sur $T_{i}$ en fonction du nombre de canaux d'un spectromètre multicanal.

effet il faut donner au calculateur un certain nombre de points expérimentaux en dehors de la raie, pour qu'il puisse en tenir compte dans la recherche de la constante représentant le fond continu. La répartition des nombres de points à l'intérieur et à l'extérieur de la raie reste à optimiser.

4. Premiers résultats expérimentaux sur une décharge récurrente. - La mesure de la température des ions a été entreprise dans la machine droite CIRCE $25 \mathrm{~kW}$. Dans celle-ci, un plasma froid d'hydrogène est injecté dans une bouteille magnétique. Il est alors chauffé à la résonance électronique par un générateur $\mathrm{H}$. $\mathrm{F}$. (puissance : $25 \mathrm{~kW}$, fréquence : $8 \mathrm{GHz}$ ) [10]. La récurrence de l'injection est de 3 cycles/s et la durée de vie $\mathrm{du}$ plasma est d'une dizaine de millisecondes. La mesure de $T_{\mathrm{i}}$ a été faite en introduisant de l'hélium en faible proportion (10 à $20 \%$ ) dans le plasma froid d'hydrogène. Le nombre de photons émis par la raie de $\mathrm{He}^{+}(\lambda=4686 \AA)$ dépend fortement des caractéristiques du plasma froid injecté.

Un volume de plasma de $3,5 \mathrm{~cm}^{3}$ vu par le spectromètre sous un angle solide de $2,5 \times 10^{-2} \mathrm{rad}$ donne pendant un cycle, sur l'ensemble des détecteurs une moyenne de 20 photons. (La transmission optique globale a été estimée à 0,2 .) Le bruit d'obscurité des photomultiplicateurs est négligeable pendant le temps d'ouverture de la porte (en moyenne : 30 impulsions/s).

La figure 8 montre l'enregistrement de la fonction instrumentale obtenu avec la raie $4713 \AA$ de l'hélium neutre. L'épaisseur du F. P. est de $0,11 \mathrm{~mm}$ soit un intervalle spectral libre de $10 \AA$. La finesse trouvée après lissage de la fonction d'Airy est de 24.

La figure 9 montre le profil de la raie $4686 \AA$ émis par le plasma, après une pose de 9 min avec une porte de $10 \mathrm{~ms}$, soit un temps de pose réel de $16 \mathrm{~s}$ environ. La température a été estimée à $40 \mathrm{eV}$. La remontée des signaux sur les canaux extérieurs est due à la présence d'une raie parasite froide (certainement d'une raie de $\mathrm{H}_{2}$ ). De plus on voit que les ailes de la raie sont noyées dans un fond continu provenant essentiellement de la lumière émise par le plasma froid. 


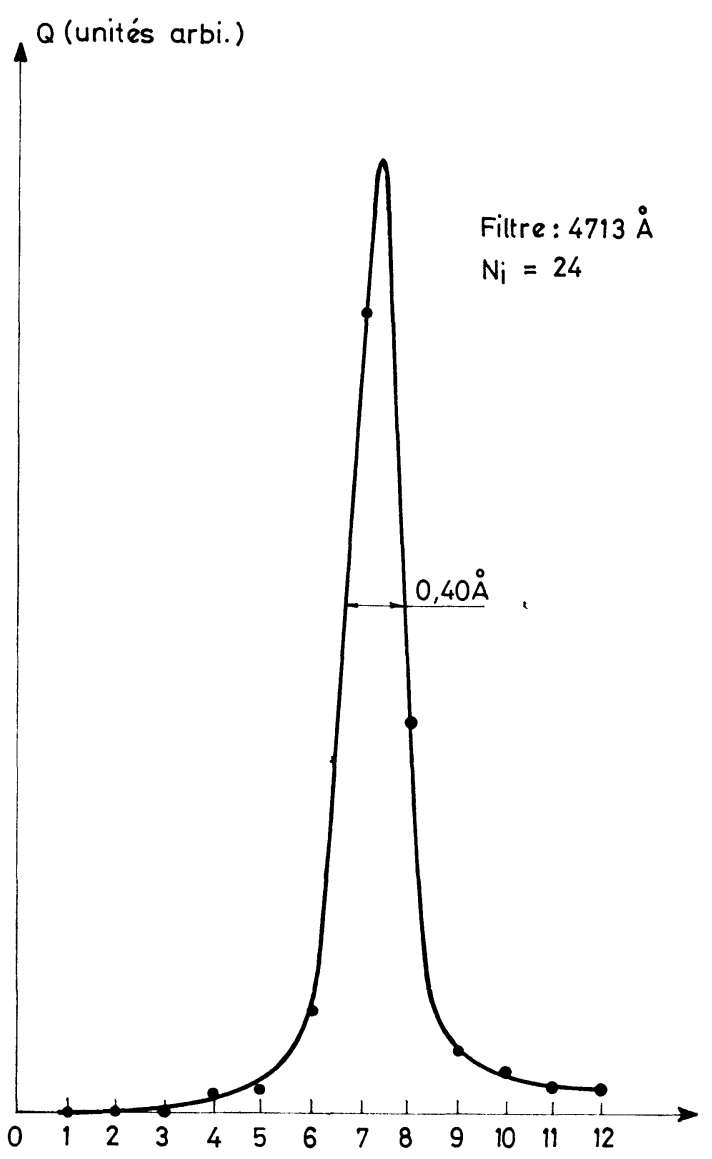

FIG. 8. - Fonction instrumentale du spectromètre mesurée avec la raie $4713 \AA$ de $\mathrm{He}$.

Des améliorations pour diminuer le taux de gaz résiduel à l'intérieur de la bouteille magnétique sont en cours actuellement. Nous pensons ainsi améliorer le contraste de la raie $4686 \AA$, et par là améliorer la précision de la mesure.

5. Conclusion. - Ces premiers résultats permettent d'affirmer que cet appareil est bien adapté au cas des plasmas récurrents peu lumineux. La luminosité de l'interféromètre Fabry-Pérot associée à la souplesse de l'électronique numérique d'acquisition a donné au spectromètre multicanal les trois qualités suivantes : sensibilité, précision, automatisme. De plus

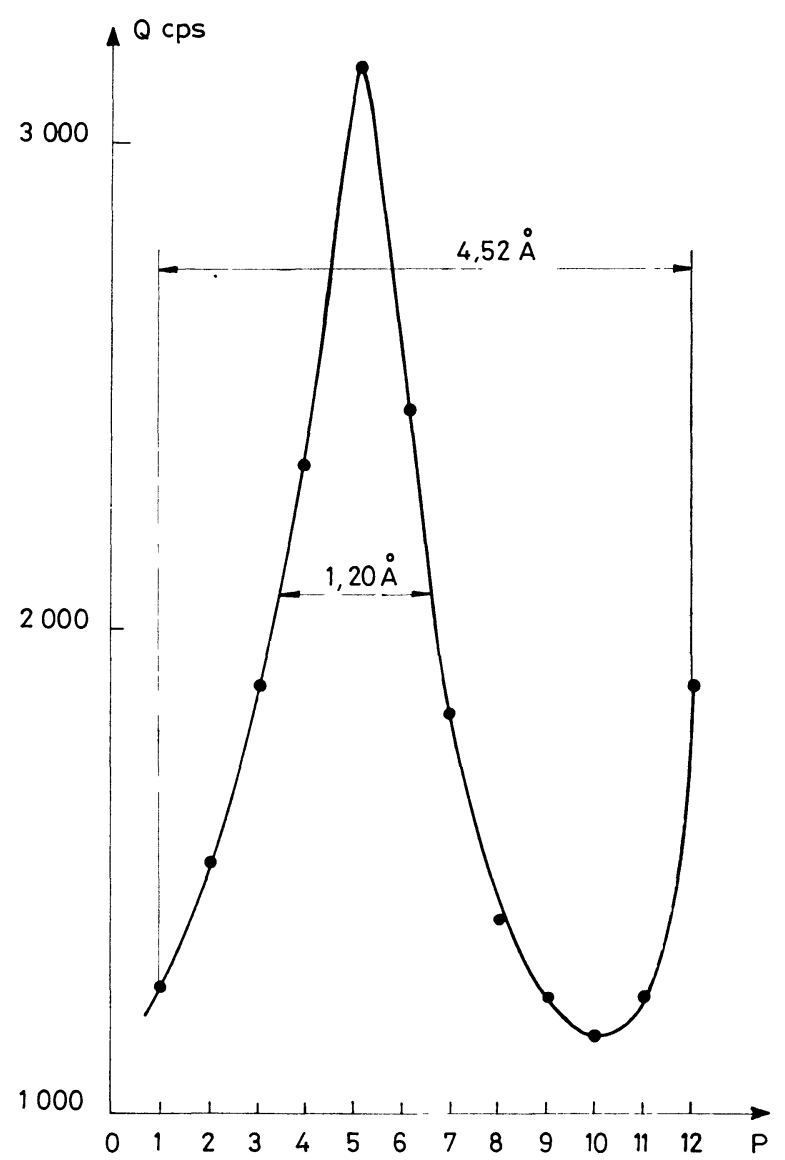

FIg. 9. - Profil de la raie $4686 \AA$ de $\mathrm{He}^{+}$enregistré sur CIRCE $25 \mathrm{~kW}$.

la stabilité de l'ensemble, les temps de pose très variables (depuis $10 \mu$ s jusqu'à plusieurs heures), la sortie sur calculatrice, et la visualisation directe des profils sont autant de points qui rendent l'appareil très fonctionnel et propre à l'étude des plasmas de longue durée de vie et peu lumineux que l'on trouve en astrophysique par exemple.

Remerciements. - Nous remercions M. Bouchareine du C. N. R. S. pour la part qu'il a pris à la réalisation optique du spectromètre multicanal digital, ainsi que M. Martin qui a collaboré au travail expérimental avec dévouement et compétence.

\section{Bibliographie}

[1] Hirschberg, J. G., Phys. Fluids 7 (1964) 543.

[2] Peacock, M. J., Cooper, J., Greig, J. R., Proc. Phys. Soc. 83 (1964) 803.

[3] Hirschberg, J. G., Platz, P., Appl. Opt. 4 (1965) 1375.

[4] Jacquinot, P., J. Opt. Soc. Am. 44 (1954) 476.

[5] Rodman, J. P., Smith, H. J., Appl. Opt. 2 (1963) 181.
[6] Tull, R. G., Appl. Opt. 7 (1968) 2023.

[7] Hays, P. B., Roble, R. G., Appl. Opt. 10 (1971) 193.

[8] Berney, A., Helv. Phys. Acta 44 (1971) 213.

[9] Clement, M., Stevenin, P., EUR-CEA-FC No 641, avril 1972.

[10] Bardet, R., Dupas, L., Gormezano, C., Melin, G., EUR$C E A-F C \mathrm{~N}^{\circ} 653,1972$. 